\title{
Role of Cytolethal Distending Toxin in Altered Stool Form and Bowel Phenotypes in a Rat Model of Post-infectious Irritable Bowel Syndrome
}

\author{
Venkata Pokkunuri, ${ }^{1}$ Mark Pimentel, ${ }^{1}$ Walter Morales, ${ }^{1}$ Sam-Ryong Jee, ${ }^{1}$ Joel Alpern, ${ }^{1}$ Stacy Weitsman, ${ }^{1}$ \\ Zachary Marsh, ${ }^{1}$ Kimberly Low, ${ }^{1}$ Laura Hwang, ${ }^{1}$ Reza Khoshini, ${ }^{1}$ Gillian M Barlow, ${ }^{1}$ Hanlin Wang ${ }^{2}$ and Christopher Chang ${ }^{1 *}$ \\ ${ }^{1}$ GI Motility Program and ${ }^{2}$ Department of Pathology, Cedars-Sinai Medical Center, Los Angeles, California, USA
}

\begin{abstract}
Background/Aims
Campylobacter jejuni infection is a leading cause of acute gastroenteritis, which is a trigger for post-infectious irritable bowel syndrome (PI-IBS). Cytolethal distending toxin (CDT) is expressed by enteric pathogens that cause PI-IBS. We used a rat model of PI-IBS to investigate the role of CDT in long-term altered stool form and bowel phenotypes.
\end{abstract}

\section{Methods}

Adult Sprague-Dawley rats were gavaged with wildtype $C$. jejuni $(C+)$, a $C$. jejuni $c d t B$ knockout (CDT-) or saline vehicle (controls). Four months after gavage, stool from 3 consecutive days was assessed for stool form and percent wet weight. Rectal tissue was analyzed for intraepithelial lymphocytes, and small intestinal tissue was stained with anti-c-kit for deep muscular plexus interstitial cells of Cajal (DMP-ICC).

Results

All 3 groups showed similar colonization and clearance parameters. Average 3-day stool dry weights were similar in all 3 groups, but day-to-day variability in stool form and stool dry weight were significantly different in the C+ group vs both controls $(P<0.01)$ and the CDT- group $(P<0.01)$, but were not different in the CDT- vs controls. Similarly, rectal lymphocytes were significantly higher after $C$. jejuni $(C+)$ infection vs both controls $(P<0.01)$ and CDT-exposed rats $(P<0.05)$. The counts in the latter 2 groups were not significantly different. Finally, c-kit staining revealed that DMP-ICC were reduced only in rats exposed to wildtype C. jejuni.

\section{Conclusions}

In this rat model of PI-IBS, CDT appears to play a role in the development of chronic altered bowel patterns, mild chronic rectal inflammation and reduction in DMP-ICC.

(J Neurogastroenterol Motil 2012;18:434-442)

Key Words

Campylobacter infections; Cytolethal distending toxin; Inflammation; Models, animal

Received: June 15, 2012 Revised: August 16, 2012 Accepted: August 21, 2012

(c) This is an Open Access article distributed under the terms of the Creative Commons Attribution Non-Commercial License (http://creativecommons. org/licenses/by-nc/3.0) which permits unrestricted non-commercial use, distribution, and reproduction in any medium, provided the original work is properly cited.

*Correspondence: Christopher Chang, MD, PhD

GI Motility Program, Cedars-Sinai Medical Center, 8730 Alden Drive, 2E, Los Angeles, CA 90048, USA

Tel: +1-310-423-6143, Fax: +1-310-423-8356, E-mail: Christopher.chang@cshs.org

Financial support: This study was supported from the Beatrice and Samuel A Seaver Foundation and the Shoolman Foundation. Conflicts of interest: None. 


\section{Introduction}

Irritable bowel syndrome (IBS) is a common gastrointestinal disorder characterized by chronic or recurrent abdominal discomfort associated with altered bowel habits, abdominal distention and bloating. ${ }^{1}$ Another characteristic feature of IBS is that symptoms alternate over time, with irregular bowel form and frequency. ${ }^{2}$ While the true pathophysiology of IBS is unknown, an increasingly recognized hypothesis is the development of IBS following an episode of acute gastroenteritis (post-infectious IBS [PI-IBS]).

While the mechanisms for the development of PI-IBS remain unknown, studies suggest that the rate of IBS development after acute gastroenteritis varies between $4 \%$ and $31 \%,{ }^{3-10}$ with 2 recent meta-analyses reporting a median incidence of $9.8 \%$ to $10 \% .^{11,12}$ In most studies, IBS was determined at least 3 months after cessation of the acute illness, and included persistent bowel disturbance with increased stool weight and increased rectal sensitivity. ${ }^{3}$ While initial criticism suggested that this was a lingering effect of the gastroenteritis, long-term studies have demonstrated persistence years later. Neal et $\mathrm{al}^{8}$ demonstrated that $57 \%$ of PI-IBS subjects continued to meet Rome criteria for IBS 6 years after the onset of PI-IBS, supporting the hypothesis that an episode of gastroenteritis could produce a persistent long-term effect.

Many bacterial pathogens can produce IBS. However, Campylobacter jejuni is the most common bacterial cause of diarrheal illness in industrialized nations. ${ }^{13}$ Moreover, acute $C$. jejuni gastroenteritis is a frequently identified antecedent to PI-IBS. ${ }^{14-16}$ Cytolethal distending toxin (CDT) is common to many human enteric bacterial pathogens. ${ }^{17}$ In $C$. jejuni, CDT is a tripartite complex of the $c d t A, c d t B$ and $c d t C$ gene products. ${ }^{18} c d t B$ has homology with DNase1-like nucleases, and is considered the active subunit. Microinjection or transfection of this subunit alone into host cells is sufficient to induce the holotoxin's effects. ${ }^{19}$ The $\mathrm{Cdt} A$ and $\mathrm{CdtC}$ subunits function in the delivery of $c d t B$ into target cells. ${ }^{18}$ Once $c d t B$ is translocated into the nucleus, it induces double-stranded DNA damage and causes arrest at the G1/S or G2/M interface of the cell cycle, depending on the cell type, ultimately leading to cell death. ${ }^{18-22}$ One in vitro study demonstrated that CDT caused irreversible cell cycle arrest of HeLa (after 24 hours) and Caco-2 cells (after 48 hours) at G2/M phase, finally leading to their death. ${ }^{20}$

In a newly developed rat model of PI-IBS, we showed that rats inoculated with $C$. jejuni develop persistent altered stool form and increased rectal intraepithelial lymphocytes (IELs) months after complete clearance of the initial infection. ${ }^{23}$ Further characterization demonstrated a reduction in deep muscular plexus interstitial cells of Cajal (DMP-ICC) in the post-infectious phase. $^{24}$ These findings are similar to findings in humans with PI-IBS. In an acute study, rats inoculated with a $C$. jejuni strain carrying an insertional deletion of $c d t B$ demonstrated mucosal damage equivalent to wildtype $C$. jejuni. ${ }^{25}$ Thus, $c d t B$ appears less important acutely. In this report, we examined the role of CDT in the development of long-term altered stool form, bowel patterns, rectal IEL elevation and DMP-ICC after clearance of $C$. jejuni infection.

\section{Materials and Methods}

\section{Campylobacter Infection of Sprague-Dawley Rats}

Adult Sprague-Dawley rats were obtained and fresh stool was cultured to establish the absence of $C$. jejuni on $\mathrm{BBL}^{\mathrm{TM}}$ Campylobacter Agar with 5 antibiotics and 10\% sheep blood (Containing the antibiotics: Amphotericin B, Cephalothin, Trimethoprim, Vancomycin and Polymyxin B; BD Diagnostics, Franklin Lakes, NJ, USA) at baseline. Prior to infection, rats received a 1 $\mathrm{mL}$ oral gavage of $5 \%$ bicarbonate solution to transiently reduce the gastric acidity. One group of rats $(\mathrm{n}=54)$ received a $1 \mathrm{~mL}$ gavage of $10^{8} \mathrm{CFU} / \mathrm{mL}$ C. jejuni 81-176 strain $(\mathrm{C}+)$. A second group of rats $(\mathrm{n}=54)$ received a $1 \mathrm{~mL}$ gavage of $10^{8} \mathrm{CFU} / \mathrm{mL}$ of a mutated $C$. jejuni 81-176 that does not express functional $c d t B$, due to an insertion of the kanamycin gene (CDT-). A third group of normal Sprague-Dawley rats $(n=25)$ were gavaged with saline vehicle only (controls). The study was approved by the Cedars-Sinai Institutional Animal Care \& Use Committee.

\section{Tracking Acute Colonization by Campyloba- cter jejuni}

After gavage, fresh stool was collected daily from infected animals. The stool specimens were cultured for the presence of $C$. jejuni on Campylobacter selective agar plates. The number of rats with at least 1 day defined successful intestinal colonization with detectable $C$. jejuni in stool. The number of days from inoculation to first detection of $C$. jejuni in the stool was then recorded to determine time to successful colonization. Stool cultures continued to be performed on a daily basis until 2 consecutive days with 
negative cultures was seen. The time from gavage to this point was marked as the clearance time.

\section{Determination of Post-infectious Phenotype}

Overall, rats were housed for 4 months. This included a 1 month post-gavage (infectious phase) and a 3-month post-infectious phase. At the end of this 4-month period, fresh stool was collected for 3 consecutive days from all rats and graded for appearance and consistency. A stool consistency score (modified Bristol Stool score) was used for grading stool. Normal stool was graded as 1; soft and poorly formed stool was graded as 2; and watery stool was graded as 3 . The collected stool was weighed and desiccated. This was accomplished by measuring stool weight before and after oven drying ( 24 hours at $70^{\circ} \mathrm{C}$ in the presence of desiccant) and percent liquid contents were calculated. After the 3-day stool collection, the rats were euthanized by $\mathrm{CO}_{2}$ asphyxiation and pneumothorax. Laparotomy was performed and the rectum was dissected, fixed in $10 \%$ formalin, imbedded in paraffin, then cut and mounted for histological evaluation as previously described. ${ }^{23}$ A section of ileum was similarly resected at a location $5 \mathrm{~cm}$ proximal to the ileocecal valve for staining of DMP-ICC as described below.

\section{Rectal Intraepithelial Lymphocytes}

After rectal tissue was fixed in formalin and sectioned for slides, sections were stained using CD3 antibody (Dako, Carpinteria, CA, USA) to visualize the presence of IEL as previously seen in humans with PI-IBS. ${ }^{14}$ At this point, all slides were randomized and coded. The slides were then examined by readers blinded to the group allocation. The reader was asked to count the number of IELs as a function of the number of epithelial cells.

\section{C-kit Staining of Deep Muscular Plexus Interstitial Cells of Cajal}

Sections of ileum from each paraffin block were stained immugnohistochemically using polyclonal rabbit anti-human CD117, c-kit (DakoCytomation, Carpinteria, CA, USA). The positive control used to test the quality of the stain was a c-kit positive gastrointestinal stromal tumor. The numbers of DMP-ICC in the entire mounted segment of ileum were counted.

\section{Statistical Methods}

During the infectious period, the colonization and clearance time were compared between the $\mathrm{C}+$ and CDT-groups using the Mann-Whitney $U$ test. At the end of 4 months (in the post-infectious phase), the average stool form was graded over 3 days and wet weight among $\mathrm{C}+, \mathrm{CDT}$ - and control rats were calculated using one-way ANOVA. Since IBS in humans is characterized by variability in stool form, we also compared the standard deviations of the stool form and wet weights between groups. The 3-day standard deviation represented the variability of the measurement. The number of rectal IEL's was reported as a ratio to the number of epithelial cells. This was also compared between the 3 groups by one-way ANOVA. Since the numbers of DMP-ICC were not normally distributed, a Mann-Whitney U test was used to compare counts between groups.

\section{Results}

\section{Campylobacter Colonization Phase}

Prior to inoculation with $C$. jejuni 81-176, C. jejuni was not detected in the stool of any of the rats at baseline. Post-gavage, 9 rats infected with $C$. jejuni ( $\mathrm{C}+$ group) died within the first 3 days after gavage ( 8 due to severe gavage trauma and 1 due to a liver tumor). The remaining rats showed no evidence of gavage-induced trauma. Thus, a total of 124 rats (45 rats in $\mathrm{C}+$ group, 54 rats in CDT-group and 25 control rats) were included for final data analysis. The number of rats with at least 1 day of detectable stool colonization by $C$. jejuni after inoculation was equal in the $\mathrm{C}+$ and CDT-groups (Fig. 1A). In the $\mathrm{C}+$ group, 38 out of 45 rats $(84 \%)$ were colonized with $C$. jejuni and in the CDT-group, 47 out of 54 rats (87\%) were colonized for at least 1 day after gavage.

Stool cultures were then followed over time. Figure 1B details and compares the colonization patterns of the 2 groups post-inoculation. The average time to first detectable $C$. jejuni in stool after gavage (colonization time) was greater in the $\mathrm{C}+$ group (6.7 \pm 4.5 days) than in the CDT-group ( $4.2 \pm 2.6$ days) $(P<0.05)$. However, when the period of time from the day of inoculation to the mean day of complete clearance of $C$. jejuni from the stool was compared between the 2 groups, it was found to be shorter in CDT- group $(10.7 \pm 4.8$ days $)$ than in the $\mathrm{C}+$ group $(12.6 \pm 5.9$ days $)(P<0.05)$. The longest period of colonization, 29 days, was observed in a rat in the $\mathrm{C}+$ group.

\section{Post-infectious Phase}

Four months after inoculation with $C$. jejuni, the 3-day average percent wet weight of stool among the groups was not sig- 
A

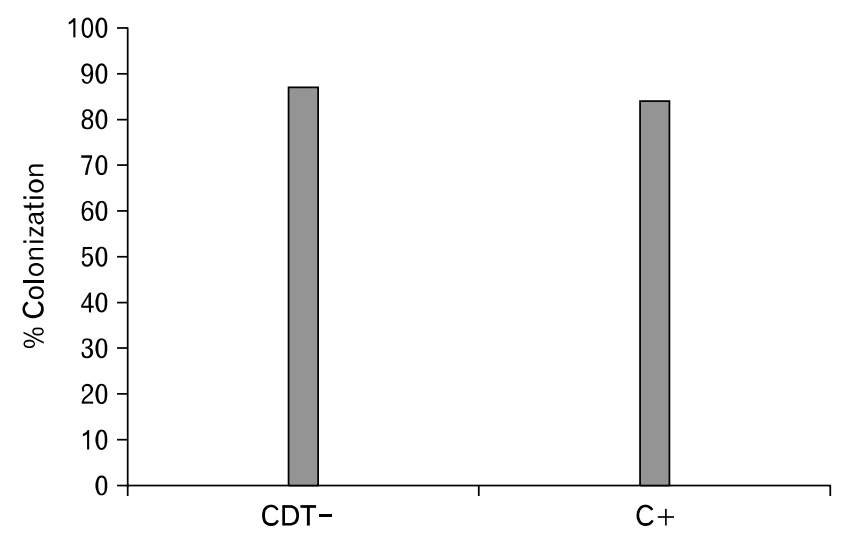

B

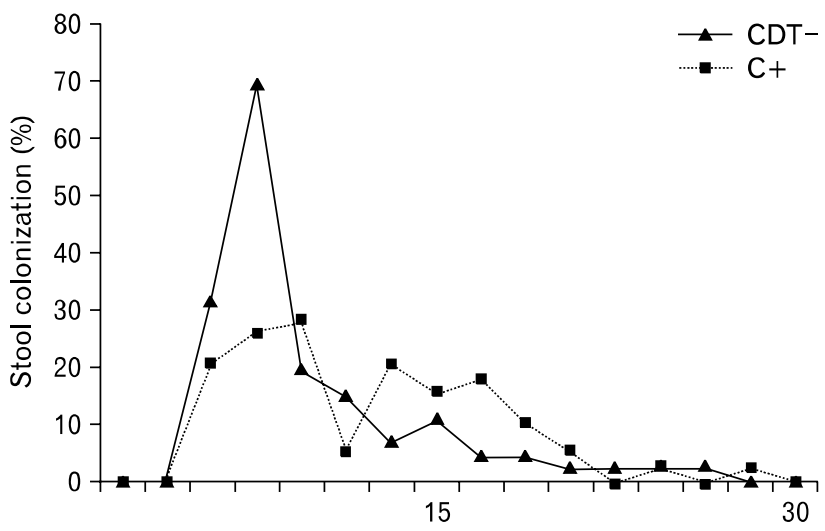

Days after inoculation

Figure 1. (A) Campylobacter colonization in rats gavaged with wildtype Campylobacter jejuni $(\mathrm{C}+)$ or a $C$. jejuni cytolethal distending toxin $\mathrm{B}(\mathrm{cdtB})$ knockout (CDT-). No significant difference was noted between C+ and CDT-groups. (B) Campylobacter colonization and clearance times in C + and CDT-groups.

A

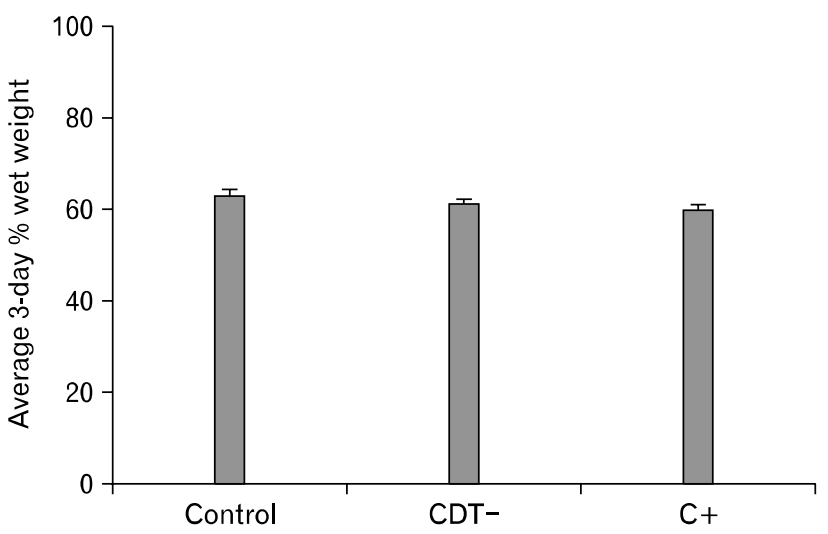

B

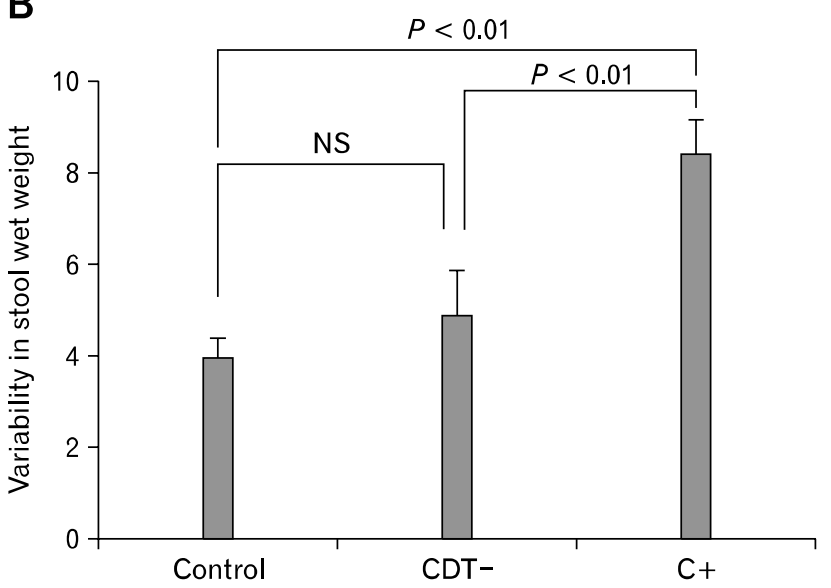

Figure 2. (A) Comparison of 3-day average stool wet weight in uninfected controls, wildtype Campylobacter jejuni $(\mathrm{C}+)$ and $C$. jejuni cytolethal distending toxin B ( $c d t B)$ knockout (CDT-) infected 4 months after gavage. No significant difference was seen between the groups. (B) Variability in stool wet weight over 3 days between groups. There was no statistical difference between control and CDT- rats.

nificantly different (Fig. 2A). However, the daily stool wet weight variability measured for 3 consecutive days demonstrated a significant difference between the groups. As shown in Figure 2B, rats in the $\mathrm{C}+$ group exhibited greater wet weight variability of stool $(8.42 \pm 0.96)$ than control rats $(3.98 \pm 0.41)(P<0.01)$ or rats in the CDT-group $(4.91 \pm 0.74)(P<0.01)$. There was no statistical difference in stool variability between control and CDT- rats.

When examining the average stool consistency (as measured based on a modified Bristol stool score) significant differences were observed between groups. From the data presented in Figure 3, the $\mathrm{C}+$ group had a higher average stool consistency (score $1.51 \pm 0.06)$ and thus less formed stool in comparison with the rats in the CDT-group $(1.23 \pm 0.03)(P<0.0001)$ and controls $(1.15 \pm 0.05)(P<0.0001)$, where 1.0 was normal. There was no statistical difference between the controls and CDT- rats.

In addition to average stool consistency, variability of stool 


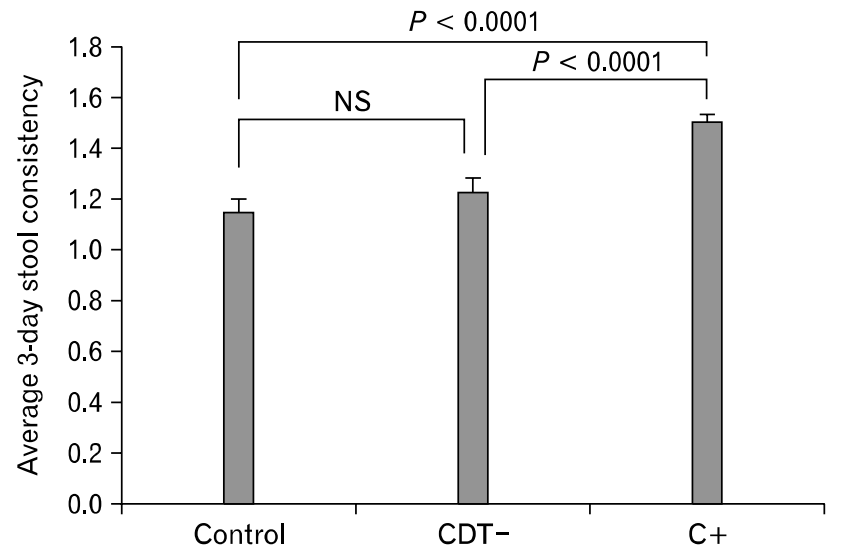

Figure 3. Average stool consistence over 3 days between groups. There was no statistical difference between control and Campylobacter jejuni cytolethal distending toxin $\mathrm{B}(c d t B)$ knockout (CDT-) rats. $\mathrm{C}+$, wildtype C. jejuni.

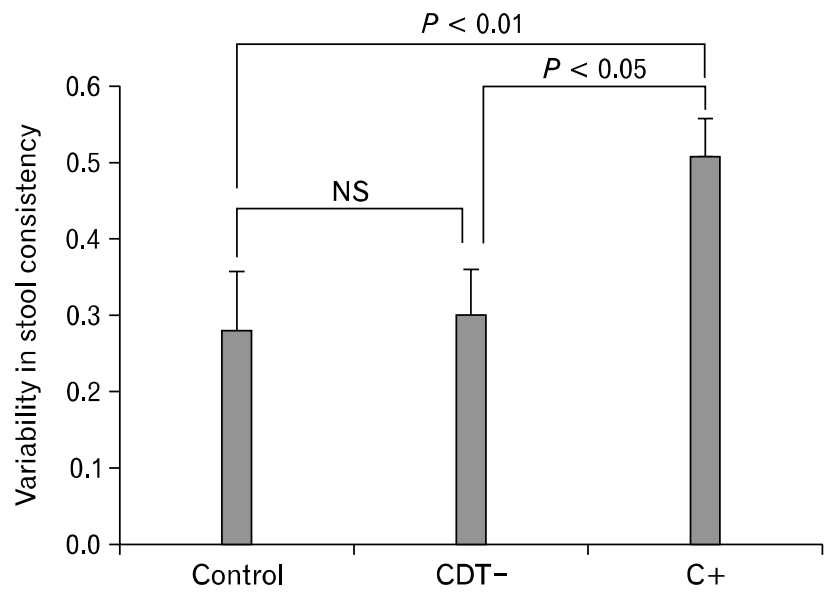

Figure 4. Variability of stool consistency over 3 days between groups. There was no statistical difference between control and CDT- rats.

Table. Quantification of Rectal Intraepithelial Lymphocytes and Deep Muscular Plexus Interstitial Cells of Cajal in Rats Gavaged With Wildtype Campylobacter jejuni, a C. jejuni Cytolethal Distending Toxin B Knockout or Saline Vehicle

\begin{tabular}{lcccc}
\hline & Controls & CDT- & C + & $P$-value \\
\hline No. of IELs ${ }^{\text {a }}$ (mean \pm SD) & $0.480 \pm 0.110$ & $0.590 \pm 0.090$ & $0.970 \pm 0.150$ & $<0.05^{\mathrm{b}}<0.01^{\mathrm{c}}$ \\
No. of DMP-ICC Controls (mean $\pm \mathrm{SD})$ & $0.218 \pm 0.159$ & $0.892 \pm 0.363$ & $0.152 \pm 0.121$ & $<0.001^{\mathrm{b}} \quad 0.024^{\mathrm{c}}$ \\
\hline
\end{tabular}

${ }^{\mathrm{a}}$ Values given denote number of intraepithelial lymphocytes per 100 epithelial cells, ${ }^{\mathrm{b}} P$-values are the difference between the control and CDT- groups, ${ }^{\mathrm{c}} P$-values are the difference between the control and $\mathrm{C}+$ groups.

CDT-, Campylobacter jejuni cytolethal distending toxin B knockout; C+, wildtype C. jejuni; IELs, intraepithelial lymphocytes; DMP-ICC, deep muscular plexus interstitial cells of Cajal.

consistency over 3 days was also compared. In this analysis, $\mathrm{C}+$ rats displayed the greatest daily variability of stool consistency $(0.51 \pm 0.06)$ compared to the variability of CDT- rats $(0.30 \pm$ $0.05)(P<0.01)$ and controls $(0.28 \pm 0.08)(P<0.05)$ (Fig. 4$)$. The CDT- rats appeared unaffected, as their stool consistency was not statistically different from the control group.

\section{Rectal Intraepithelial Lymphocytes}

Differences were also seen in the number of rectal IEL in the 3 groups. The number of rectal IELs was highest in $\mathrm{C}+$ rats $(0.97 \pm 0.15 / 100$ epithelial cells) compared to controls $(0.48 \pm$ 0.11 per 100 epithelial cells, Table $)(P<0.01)$ and CDT-rats $(0.59 \pm 0.09 / 100$ epithelial cells, Table $)(P<0.05)$ (Fig. 5). As with previous results, the CDT- rats were not statistically different from controls.

\section{Deep Muscular Plexus Interstitial Cells of Cajal}

The DMP-ICC staining from the 3 groups was compared.
In each case, the numbers of DMP-ICC in the entire mounted segment of ileum were counted. Months after clearance of infection, $\mathrm{C}+$ rats had a noticeable and consistent reduction in DMP-ICC $(0.152 \pm 0.121)$ (Fig. 6C) when compared to controls $(0.218 \pm 0.159$, Table $)(P=0.024$, Fig. 6A). However in CDT- rats, DMP-ICC numbers were not only preserved but greater in number than in controls $(0.892 \pm 0.363$, Table $)(P<$ 0.001 compared both to controls and to $\mathrm{C}+$, Fig. $6 \mathrm{~B}$ ).

\section{Discussion}

The results of this study demonstrate that rats inoculated with wildtype $C$. jejuni 81-176 (C+ rats) exhibit altered variability of stool form and wet weight 4 months after infection. In contrast, rats inoculated with a mutant $C$. jejuni strain that does not express $c d t B$ (CDT- rats) exhibited bowel patterns that were closer to normal, as determined from uninfected control rats. In addition, rats exposed to wildtype $C$. jejuni demonstrated elevated rectal IELs and reduction in DMP-ICC, which was not seen in 

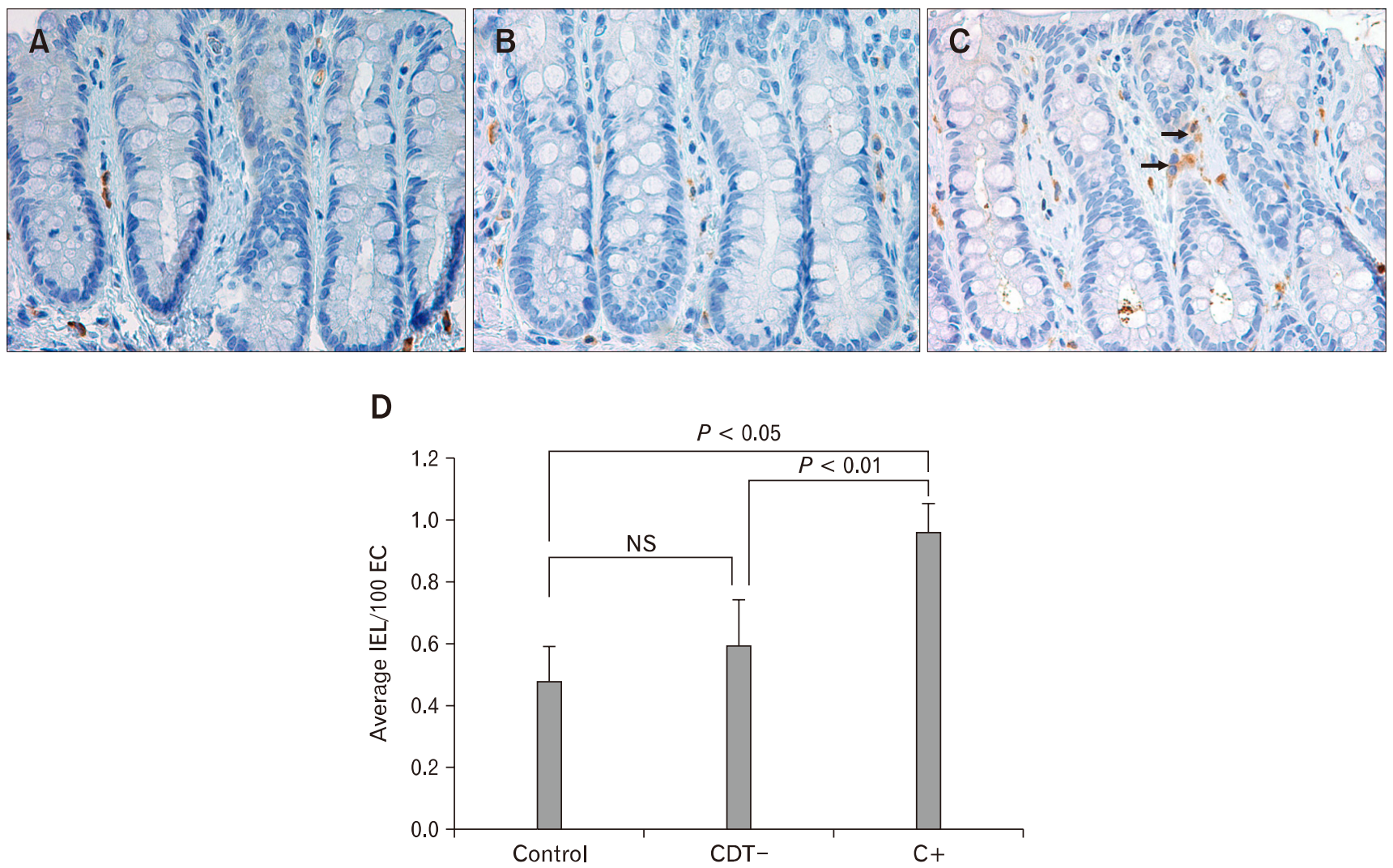

Figure 5. Immunohistochemical staining of rectal tissue from control (A), Campylobacter jejuni cytolethal distending toxin B knockout (CDT-, B) and wildtype $C$. jejuni $(\mathrm{C}+, \mathrm{C})$ rats with antibodies to $\mathrm{CD} 3$, to evaluate rectal intraepithelial lymphocytes. (D) Comparison of average intraepithelial rectal lymphocytes (per 100 epithelial cells) in uninfected controls, C+ and CDT- infected rats. There was no statistical difference between control and CDTrats. IEL, intraepithelial lymphocytes; EC, epithelial cell.
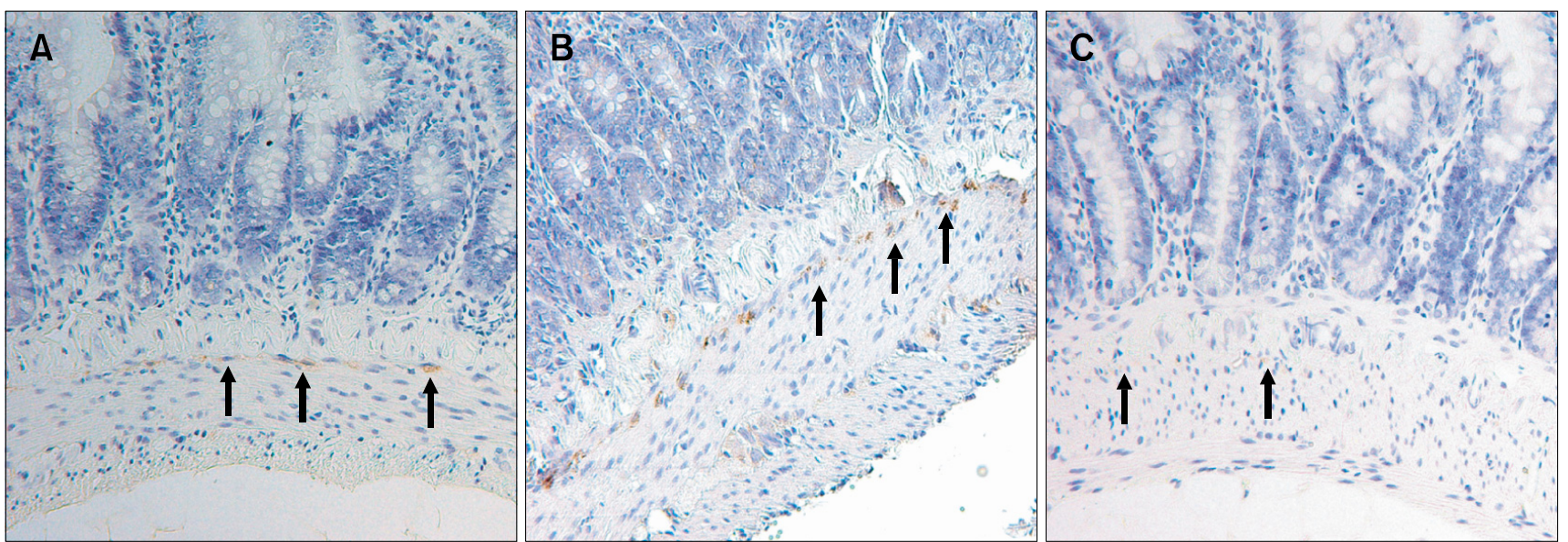

Figure 6. Immunohistochemical staining of ileal tissue from control (A), Campylobacter jejuni cytolethal distending toxin B knockout (CDT-, B) and wildtype $C$. jejuni $(\mathrm{C}+, \mathrm{C})$ rats with $\mathrm{CD} 117$ to evaluate deep muscular plexus interstitial cells of Cajal ( $\times 20$ magnification).

rats inoculated with the CDT- $C$. jejuni. In this PI-IBS rat model, 3 months after complete clearance of $C$. jejuni infection, CDT appears to be a determinant of the development of altered stool form, irregular bowel pattern and inflammation, but does not appear to affect the establishment of initial colonization during acute infection. 
Development of IBS has been demonstrated in patients infected with numerous bacterial pathogens including Shigella, Escherichia coli and Salmonella. However, development of PI-IBS has been most thoroughly studied in patients who were infected with $C$. jejuni. ${ }^{3-10}$ The strongest risk factor for the development of PI-IBS appears to be prolonged duration of gastroenteritis (relative risk $=11.5) .{ }^{4}$ Human subjects with PI-IBS display the characteristic features of IBS, including altered stool form. ${ }^{2}$ Another physiologic hallmark of PI-IBS is increased rectal IEL counts. Spiller et $\mathrm{al}^{14}$ have demonstrated that 1 year after Campylobacter infection, the number of enteroendochrine cells and IELs (per 100 epithelial cells) in the rectum of PI-IBS patients remained significantly elevated compared to a control group $(P<$ $0.001)^{14}$

While animal models of PI-IBS are limited, there is a well-characterized model in a mouse previously infected with the parasitic pathogen Trichenella spiralis. ${ }^{26}$ In this model the focus has been on post-infectious gut neuromuscular dysfunction and visceral hyperalgesia. Although a human outbreak of Trichenella britovi from Turkey has demonstrated an overall $13.9 \%$ incidence of IBS following infection, this pathogen is not common in western countries. ${ }^{27}$

In a recent study, we demonstrated that rats exposed to $C$. jejuni had greater altered stool form. ${ }^{23}$ Additionally, these rats demonstrated increased IEL counts in the rectum and left colon, but not in cecum or small bowel. This new rat model reproduces IBS phenotypes, including altered stool form and increased rectal lymphocytes.

Various lines of evidence suggest that during C. jejuni infection, CDT is a key virulent factor. Using a severe combined immunodeficient mouse model, Purdy et $\mathrm{al}^{28}$ demonstrated greater tissue (blood and liver) invasion and virulence by wildtype $C$. jejuni expressing CDT compared to $C$. jejuni CDT mutants, although the intestinal colonization levels remained equal in both 2 groups. This observation was further corroborated with a limited enteric flora mouse infection model. ${ }^{29}$ Fox et $\mathrm{al}^{30}$ demonstrated that $C$. jejuni $c d t B$ mutants failed to colonize the gastrointestinal tract of $\mathrm{C} 57 \mathrm{BL} / 129$ mice (immune competent) in contrast to wildtype C. jejuni ( $0 \%$ vs $50 \%$ ). In addition, using nuclear factor $\kappa \mathrm{B}$-deficient mice $(3 \mathrm{X})$, they also reported that, despite persistent and equal gastrointestinal colonization rates (100\%) $3 \mathrm{X}$ mice infected with wildtype $C$. jejuni exhibited severe gastrointestinal inflammatory changes, compared to modest gastrointestinal inflammatory changes in mice infected with a $c d t B$ mutant. These results imply that CDT may contribute to persis- tent gastrointestinal colonization, by playing a role in the ability of C. jejuni to escape immune surveillance in immunocompetent C57BL/129 mice (but not in 3X mice). One proposed mechanism for this phenomenon is its ability to cause cell cycle arrest of lymphocytes at G2/M phase, similar to its effect in epithelial cells. ${ }^{31}$ Also, less severe inflammation in $3 \mathrm{X}$ mice suggests that inflammation of the gastrointestinal tract is a feature of CDT activity. ${ }^{30}$ More recently, Jain et $\mathrm{al}^{32}$ demonstrated that suckling mice (which lack a fully mature immune system) inoculated with $c d t B+C$. jejuni developed pan-mural inflammation with mucosal denudation and necrosis affecting the jejunum, ileum and colon in all mice, compared to mice infected with $c d t B-C$. jejuni, which showed mild inflammatory changes only in the descending colon. These findings are consistent with in vitro studies reporting that CDT induces IL-8 from intestinal cell line INT407. ${ }^{33}$

Our current study supports a longer colonization time among rats infected with wildtype $C$. jejuni vs CDT- $C$. jejuni. However, in a recently published study of acute histological changes in rats exposed to wildtype and CDT- C. jejuni, there very few histological differences during the acute infection during first 4 weeks after gavage. ${ }^{25}$ Both groups had significant disturbances including epithelial cell ballooning, villous blunting and villous tip disruption in the absence of cellular inflammation; these changes resolved by 3 months post-infection. Based on these findings, the role of CDT may involve mechanisms that are not related to the events surrounding the initial acute infection.

Various mechanisms of CDT action have been reported. For example, CDT may cause growth arrest of villous epithelial cells, thereby decreasing the barrier function of the intestinal epithelium and producing loss of nutrient absorption. ${ }^{20}$ It is also possible that cell cycle arrest of the intestinal epithelial cells would increase the contact time between the host and the pathogen. ${ }^{34}$ Whether CDT-deficient strains of $C$. jejuni produce less severe diarrheal disease and other chronic sequelae in human infections remains to be assessed directly. Many studies have reported the existence of higher frequency of $c d t B+C$. jejuni compared to $c d t B$ - strains, ${ }^{32,35-37}$ signifying that $c d t B$ is present in majority of $C$. jejuni strains. In a recently concluded study, Jain et al demonstrated that $c d t B+C$. jejuni strains adhere to and invade the HeLa cells, more effectively than the $c d t B$ - strains. ${ }^{32} C$. jejuni and other CDT-producing enteric pathogens may have effects on intestinal epithelial cells; specifically cells in the intestinal crypts resulting in rapid G2 phase cell cycle arrest of proliferating cells leading to their growth arrest. ${ }^{38}$ Thus, CDT may have profound effects on crypt cell maturation into functional villous epithelial 
cells. This effect may also be responsible for a temporary villous epithelial erosion and loss of absorptive functions, ${ }^{39-41}$ as suggested by 2 in vitro studies that reported elongation and irreversible loss of tissue culture cells (without recovery of the affected cells) at the end of 72 hours when treated with $C$. jejuni CDT. $^{42,43}$

The mechanism linking these in vitro toxic effects to prolonged bowel disturbance is uncertain. However, increases in inflammatory and enteroendocrine cells, observed in colonic mucosa of PI-IBS patients, could result in altered bowel patterns and changes in neuromuscular function. ${ }^{14}$ One interesting study showed that Campylobacter enteritis leads to loss of gut mucosal neural staining. ${ }^{44}$ Enteric neuronal damage and subsequent bowel dysfunction may result from immune mediated mechanisms that disturb the enteric nervous system. Our results support this possibility. In our study, CDT-C. jejuni did not result in loss of DMP-ICC, which suggests that CDT may have a role in degeneration of neuronal function in PI-IBS. Another notable example of neurotoxicity is Campylobacter-induced post-infective polyneuritis, known as Guillain-Barré syndrome, in which Campylobacter, through molecular mimicry, induces a host immune response against the GM1 ganglioside in peripheral nerves. ${ }^{45}$

Modest elevations in rectal lymphocytes are a sign of chronic inflammation and have been shown in a number of human studies of PI-IBS patients. Spiller and colleagues ${ }^{14}$ measured an approximately 2-fold increase in IELs per 100 epithelial cells in PI-IBS subjects approximately 12 weeks after acute gastroenteritis, versus healthy controls ( 0.9 vs $0.5, P$-value NS). Gwee et $a^{5}$ quantified the total number of mononuclear cells per high power field (HPF). Chronic inflammatory cells rose from $79.4 / \mathrm{HPF}$ to 105.7/HPF $(P<0.01)$ in rectal biopsies from PI-IBS patients 3 months post-infection, compared to healthy controls. Comparable increases in lamina propria $\mathrm{T}$-cells were measured by Dunlop et al. ${ }^{46}$ Results from this post-infectious rat study demonstrate a comparable, significant 2-fold rise in rectal IELs 4 months after C. jejuni infection, compared to uninfected controls. Unlike human studies, all infected rats were categorized as post-infectious and analyzed regardless of the degree of "IBS-like" phenotypes.

In this study, CDT appears important in the development of altered bowel function and increased rectal IELs in our PI-IBS rat model. The stool form and its variability were significantly closer to normal among rats infected with a strain that failed to express $c d t B$. It is unclear whether CDT is the sole agent in determining the development of this altered stool form or if any of the previously described mechanisms is responsible for these features. Perhaps CDT has a neurotoxic effect. This intriguing possibility is supported by our recently published finding that wildtype CDT produces a reduction in DMP-ICC in this rat model. $^{24}$ In contrast, CDT- rats showed DMP-ICC staining that was not reduced but increased compared to controls. This increase in ICC could be a response to infection in the absence of CDT; lack of growth arrest of ICCs or lack of cross-reacting immune response to ICCs may underlie this finding, but more work is needed to determine whether these or other mechanisms explain this observation. Future studies need to evaluate the effect of CDT on the gut neuromuscular apparatus.

In conclusion, using wild type and CDT- strains of $C$. jejuni in a post-infectious IBS rat model, we demonstrate that Campylobacter CDT is an important factor in the development of altered stool form, chronic altered bowel pattern, rectal inflammation and reduced DMP-ICC, since the absence of this toxin resulted in post-infectious phenotypes similar to those in uninfected control rats.

\section{References}

1. Thompson WG, Longstreth GF, Drossman DA, Heaton KW, Irvine EJ, Müller-Lissner SA. Functional bowel disorders and functional abdominal pain. Gut 1999;45(suppl 2):II43-II47.

2. Pimentel M, Hwang L, Melmed GY, et al. New clinical method for distinguishing D-IBS from other gastrointestinal conditions causing diarrhea: the LA/IBS diagnostic strategy. Dig Dis Sci 2010;55: 145-149.

3. McKendrick MW, Read NW. Irritable bowel syndrome - post salmonella infection. J Infect 1994;29:1-3.

4. Neal KR, Hebden J, Spiller R. Prevalence of gastrointestinal symptoms six months after bacterial gastroenteritis and risk factors for development of the irritable bowel syndrome: postal survey of patients. BMJ 1997;314:779-782.

5. Gwee KA, Leong YL, Graham C, et al. The role of psychological and biological factors in postinfective gut dysfunction. Gut 1999;44: 400-406.

6. Parry SD, Stansfield R, Jelley D, et al. Is irritable bowel syndrome more common in patients presenting with bacterial gastroenteritis? A community-based, case-control study. Am J Gastroenterol 2003;98: 327-331.

7. Barber R, Blakey A. Prevalence of gastrointestinal symptoms after bacterial gastroenteritis. Study did not include a control group. BMJ 1997;314:1903

8. Neal KR, Barker L, Spiller RC. Prognosis in post-infective irritable bowel syndrome: a six year follow up study. Gut 2002;51:410-413.

9. Okhuysen PC, Jiang ZD, Carlin L, Forbes C, DuPont HL. Post-diarrhea chronic intestinal symptoms and irritable bowel syndrome in North American travelers to Mexico. Am J Gastroenterol 2004;99:1774-1778.

10. Mearin F, Pérez-Oliveras M, Perello A, et al. Dyspepsia and irritable bowel syndrome after a Salmonella gastroenteritis outbreak: one-year follow-up cohort study. Gastroenterology 2005;129:98-104. 
11. Halvorson HA, Schlett CD, Riddle MS. Postinfectious irritable bowel syndrome - a meta-analysis. Am J Gastroenterol 2006;101: 1894-1899.

12. Thabane M, Kottachchi DT, Marshall JK. Systematic review and meta-analysis: The incidence and prognosis of post-infectious irritable bowel syndrome. Aliment Pharmacol Ther 2007;26:535-544.

13. Friedman J NJ, Wegener HC, Tauxe RV. Epidemiology of Campylobacter jejuni infections in the United States and other industrialized nations. In: Nachamkin I BM, ed. Campylobacter. 2 ed. Washington: American Society for Microbiology 2000:121-138.

14. Spiller RC, Jenkins D, Thornley JP, et al. Increased rectal mucosal enteroendocrine cells, $\mathrm{T}$ lymphocytes, and increased gut permeability following acute Campylobacter enteritis and in post-dysenteric irritable bowel syndrome. Gut 2000;47:804-811.

15. Mead PS, Slutsker L, Griffin PM, Tauxe RV. Food-related illness and death in the United States reply to Dr. Hedberg. Emerg Infect Dis 1999;5:841-842.

16. Allos BM. Campylobacter jejuni infections: update on emerging issues and trends. Clin Infect Dis 2001;32:1201-1206.

17. Pickett CL, Whitehouse CA. The cytolethal distending toxin family. Trends Microbiol 1999;7:292-297.

18. Lara-Tejero M, Galán JE. CdtA, CdtB, and $\mathrm{CdtC}$ form a tripartite complex that is required for cytolethal distending toxin activity. Infect Immun 2001;69:4358-4365.

19. Lara-Tejero M, Galán JE. A bacterial toxin that controls cell cycle progression as a deoxyribonuclease I-like protein. Science 2000;290: 354-357.

20. Whitehouse CA, Balbo PB, Pesci EC, Cottle DL, Mirabito PM, Pickett CL. Campylobacter jejuni cytolethal distending toxin causes a G2-phase cell cycle block. Infect Immun 1998;66:1934-1940.

21. Hassane DC, Lee RB, Pickett CL. Campylobacter jejuni cytolethal distending toxin promotes DNA repair responses in normal human cells. Infect Immun 2003;71:541-545.

22. Hassane DC, Lee RB, Mendenhall MD, Pickett CL. Cytolethal distending toxin demonstrates genotoxic activity in a yeast model. Infect Immun 2001;69:5752-5759.

23. Pimentel $\mathrm{M}$, Chatterjee $\mathrm{S}$, Chang $\mathrm{C}$, et al. A new rat model links two contemporary theories in irritable bowel syndrome. Dig Dis Sci 2008;53:982-989.

24. Jee SR, Morales W, Low K, et al. ICC density predicts bacterial overgrowth in a rat model of post-infectious IBS. World J Gastroenterol 2010;16:3680-3686.

25. Morales W, Pimentel M, Hwang L, et al. Acute and chronic histological changes of the small bowel secondary to $C$. jejuni infection in a rat model for post-infectious IBS. Dig Dis Sci 2011;56:2575-2584.

26. Bercík P, Wang L, Verdú EF, et al. Visceral hyperalgesia and intestinal dysmotility in a mouse model of postinfective gut dysfunction. Gastroenterology 2004;127:179-187.

27. Soyturk M, Akpinar H, Gurler O, et al. Irritable bowel syndrome in persons who acquired trichinellosis. Am J Gastroenterol 2007;102: 1064-1069.

28. Purdy D, Buswell CM, Hodgson AE, McAlpine K, Henderson I, Leach SA. Characterisation of cytolethal distending toxin (CDT) mutants of Campylobacter jejuni. J Med Microbiol 2000;49:473-479.
29. Chang C, Miller JF. Campylobacter jejuni colonization of mice with limited enteric flora. Infect Immun 2006;74:5261-5271.

30. Fox JG, Rogers AB, Whary MT, et al. Gastroenteritis in NF-kappaB-deficient mice is produced with wild-type Camplyobacter jejuni but not with C. jejuni lacking cytolethal distending toxin despite persistent colonization with both strains. Infect Immun 2004;72: 1116-1125.

31. Gelfanova V, Hansen EJ, Spinola SM. Cytolethal distending toxin of Haemophilus ducreyi induces apoptotic death of Jurkat T cells. Infect Immun 1999;67:6394-6402.

32. Jain D, Prasad KN, Sinha S, Husain N. Differences in virulence attributes between cytolethal distending toxin positive and negative Campylobacter jejuni strains. J Med Microbiol 2008;57:267-272.

33. Hickey TE, McVeigh AL, Scott DA, et al. Campylobacter jejuni cytolethal distending toxin mediates release of interleukin-8 from intestinal epithelial cells. Infect Immun 2000;68:6535-6541.

34. Lara-Tejero M, Galán JE. Cytolethal distending toxin: limited damage as a strategy to modulate cellular functions. Trends Microbiol 2002;10:147-152.

35. Bang DD, Scheutz F, Ahrens P, Pedersen K, Blom J, Madsen M. Prevalence of cytolethal distending toxin (cdt) genes and CDT production in Campylobacter spp. isolated from Danish broilers. J Med Microbiol 2001;50:1087-1094.

36. Dassanayake RP, Zhou Y, Hinkley S, et al. Characterization of cytolethal distending toxin of Campylobacter species isolated from captive macaque monkeys. J Clin Microbiol 2005;43:641-649.

37. Al-Mahmeed A, Senok AC, Ismaeel AY, Bindayna KM, Tabbara KS, Botta GA. Clinical relevance of virulence genes in Campylobacter jejuni isolates in Bahrain. J Med Microbiol 2006;55:839-843.

38. Walker RI, Caldwell MB, Lee EC, Guerry P, Trust TJ, RuizPalacios GM. Pathophysiology of Campylobacter enteritis. Microbiol Rev 1986;50:81-94.

39. Gordon JI. Intestinal epithelial differentiation: new insights from chimeric and transgenic mice. J Cell Biol 1989;108:1187-1194.

40. Ketley JM. Pathogenesis of enteric infection by Campylobacter. Microbiology 1997;143(Pt 1):5-21.

41. Sears CL, Kaper JB. Enteric bacterial toxins: mechanisms of action and linkage to intestinal secretion. Microbiol Rev 1996;60:167-215.

42. Johnson WM, Lior H. Cytotoxic and cytotonic factors produced by Campylobacter jejuni, Campylobacter coli, and Campylobacter laridis. J Clin Microbiol 1986;24:275-281.

43. Johnson WM, Lior H. A new heat-labile cytolethal distending toxin (CLDT) produced by Campylobacter spp. Microb Pathog 1988;4: 115-126.

44. Jenkins D, Thornley JP, Wright T, et al. Enterochromaffin \& mast cell hyperplasia parallels mucosal nerve damage following Campylobacter enteritis [abstract]. Neurogastroenterol Motil 1998;10:464.

45. Oomes PG, Jacobs BC, Hazenberg MP, Bänffer JR, van der Meché FG. Anti-GM1 IgG antibodies and Campylobacter bacteria in GuillainBarre syndrome: evidence of molecular mimicry. Ann Neurol 1995; 38:170-175.

46. Dunlop SP, Jenkins D, Neal KR, Spiller RC. Relative importance of enterochromaffin cell hyperplasia, anxiety, and depression in postinfectious IBS. Gastroenterology 2003;125:1651-1659. 